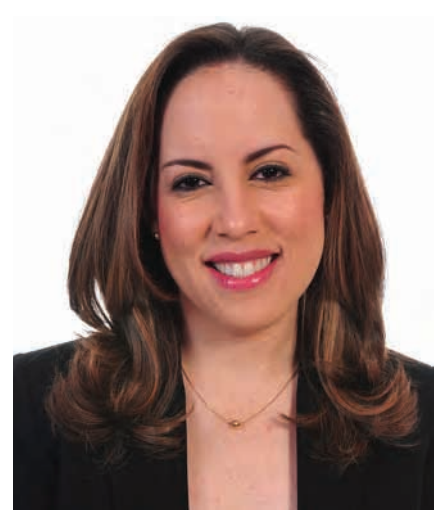

VENTANAS ABIERTAS A LA PEDAGOGÍA UNIVERSITARIA

\title{
Investigación sobre la práctica docente en las universidades
}

\section{Martha Báez}

Resumen: La investigación sobre la práctica docente (ISPD) en el contexto universitario es un estudio crítico de la docencia que busca reflexionar sobre esta para mejorar su incidencia en la calidad del aprendizaje de los estudiantes. Este artículo se basa en la experiencia de académicos anglosajones con este tipo de investigaciones, abordando los fundamentos conceptuales y metodológicos que la caracterizan y su importancia para el desarrollo individual e institucional de la docencia. Sin pretender hacer una presentación comparativa, se muestran elementos que asemejan y distancian la ISPD de la investigación tradicional.

Abstract: Scholarship of Teaching and Learning (SoTL) in the university context is a critical study of teaching aimed at reflecting on its impact improving the quality of student learning. This article is based on the experience of Anglo-Saxon scholars on this type of study. It addresses the conceptual and methodological characteristics of this practice and its importance to the individual and institutional development of the teaching practice. Elements that relate and distant the SoTL from the traditions research are shown.

Desde que se inició la Especialidad en Pedagogía Universitaria en la Pontificia Universidad Católica Madre y Maestra, se ha estado profundizando en la investigación sobre la práctica docente (ISPD). Este es el nombre en español con el que se propone acuñar, en la PUCMM, el llamado Scholarship of Teaching and Learning (SoTL), un tipo de investigación llevada a cabo en las universidades con la intención de hacer una reflexión crítica de nuestra docencia. La ISPD se fundamenta en evidencias y se realiza con el propósito de mejorar la propia docencia. Es un estilo de investigación que implica un ejercicio individual y colectivo, orientado a la reflexión para la construcción de una conciencia colectiva de nuestra docencia y que tiene como fin último garantizar la calidad del aprendizaje de los estudiantes.

En este artículo se busca contribuir a la comprensión de la ISPD haciendo énfasis en su significado y los propósitos que persigue, los tipos de preguntas de investigación que se plantea, los métodos que utiliza, el rol que juega el investigador y las implicaciones de esta práctica en la política universitaria. Aunque el artículo no pretende hacer una comparación de la ISPD con la investigación tradicional, en él se abordan algunas de las críticas más importantes que alejan la ISPD de su validez como ejercicio científico.

\section{¿De qué se trata la Investigación sobre la Práctica Docente (ISPD)?}

La mayor fuente de información de la ISPD la encontramos en la literatura anglosajona, proveniente principalmente, pero no exclusivamente, de Estados Unidos, Canadá, Reino Unido y Australia. En esta literatura se relaciona la ISPD con calificativos tan variados como Scholarship of Teaching and $L$ earning (SoTL), practitioner inquiry, self-study, critical action research, collaborative tearcher inquiry, entre otros calificativos en el idioma inglés. Aunque los anteriores no son precisamente apelativos intercambiables, todos tienen en común su referencia a un género de la investigación educativa en la que, como indican Conchran-Smith y Donnell (2006), el practicante es el mismo investigador, el contexto profesional es el lugar de la investigación y la práctica en sí misma es el foco de estudio.

\section{Key Words}

scholarship of teaching and learning (SoTL), educational research, university teaching policy.

\begin{abstract}
* Magíster en Desarrollo Internacional por Brandeis University, Massachusetts, Estados Unidos, y Especialista en Estudios Internacionales e Interdisciplinarios en Población y Desarrollo Sostenible por la Universidad de Chile. Encargada de la Unidad de Investigación de la Vicerrectoría de Investigación de la Pontificia Universidad Católica Madre y Maestra. Profesora Instructora de esa misma Universidad. Para contactar a la autora: mbaez@pucmmsti.edu.do.
\end{abstract}

Encuentre el texto en "La Investigación sobre la Práctica Docente", http://www.pucmm.edu.do/STI/campus/CDP/ComuncacionPublicaciones/Paginas/CuadernodePedagogiaUniversitaria.aspx

Cuaderno de Pedagogía Universitaria Año 9/ N. 17 /enero-junio 2012 / Santiago, Rep. Dom.: PUCMM/p. 3-8

\section{Palabras clave}

docente, investigación educativa, politica docente 
A partir de las denominaciones de investigación antes mencionadas, parecería que nos estamos enfrentando a un paradigma de investigación ya conocido, debido a que algunas de estas prácticas de estudio forman parte de la tradición investigativa en el área educativa. Sin embargo, la ISPD es considerada como un fenómeno de estudio relativamente reciente. Se refiere más directamente al calificativo en inglés scholarship of teaching and learning (SoTL), que es un proceso de aprendizaje continuo sobre las prácticas de la propia enseñanza que requiere de una demostración pública de dicho conocimiento con el fin último de mejorar la calidad del aprendizaje de los estudiantes (McKinney, s.f.).

La Fundación Carnegie, un centro independiente de políticas e investigación educativa, líder en el estudio de SoTL en Estados Unidos, la define como un ejercicio que implica el planteamiento de problemas acerca de un tema relacionado con la enseñanza, o el aprendizaje, el estudio del problema mediante métodos adecuados a las epistemologías disciplinares, la aplicación de los resultados a la práctica, la comunicación de los resultados, la auto-reflexión y la revisión entre pares (Cambridge, 2004). En estas aproximaciones a la ISPD, la motivación ulterior por mejorar el aprendizaje de los estudiantes nos lleva a cuestionar nuestro propio quehacer docente e ir tras evidencias para superarlo. La transformación viene dada entonces por nuestra reflexión crítica de las evidencias recolectadas. En este sentido, más que la generación de un conocimiento que pueda ser generalizable, lo que se busca con la ISPD es mejorar la calidad del aprendizaje de los estudiantes dentro de su propio contexto y con los recursos específicos con los que se cuenta.

El autor más influyente en el surgimiento de esta práctica es Ernest Boyer (Caster \& Hautala, 2008; Cochran-Smith \& Donnell, 2006; Proseer, 2008; Shulman, 2011). Los análisis del ensayo que escribió en 1990, en el que Boyer presenta un reporte sobre las prioridades del profesorado (Scholarship Reconsidered: Priorities of the Professoriate), es considerado como el trabajo detonante del fenómeno de la ISPD en la educación superior. Según reporta Prosser (2008), en este trabajo Boyer hace un análisis distintivo entre la investigación tradicional orientada al descubrimiento, la revisión de la literatura que persigue la integración de conocimientos, la investigación aplicada que se relaciona con el estudio de problemas locales a los que se les busca una solución práctica y la investigación sobre la práctica docente que se relaciona con una reflexión crítica de la práctica docente basada en evidencias para mejorar la propia práctica.

Aunque lo que llamamos ISPD es presentada por Boyer como un género de investigación distinto a los tradicionales, ésta comparte con ellos algunas características. Una de las más destacadas es su búsqueda por hacerse pública, lo que implica la posibilidad de ser revisada de manera crítica por los miembros de la comunidad académica a la que se relaciona y la posibilidad de ser construida sobre los avances que otros han logrado en el campo en cuestión (Shulman, 2011). En este sentido, al igual que cualquier otra investigación, la ISPD persigue convertirse en un bien de propiedad comunitaria que busca su aceptación a través de la revisión de los pares.
Otro de los autores que destacan tanto la dimensión de propiedad comunitaria de la ISPD como su distancia de la investigación tradicional es Michael Prosser, un académico de la Universidad de Hong Kong. Prosser (2008) afirma que la ISPD no es una investigación en el sentido tradicional, indicando que se trata de una actividad de orientación práctica, llevada a cabo colegiadamente, y cada vez más conducida junto con la investigación tradicional dentro de las disciplinas.

La intencionalidad de la ISPD parece estar clara, no obstante, su definición y conceptualización es considerada en construcción, debido a lo novedoso de esta práctica. La apuesta de los que promueven este fenómeno es a que el mismo se vaya enriqueciendo con la propia experiencia de los académicos que se dedican a practicarla.

\section{La pregunta de investigación}

En la ISPD, como en la investigación tradicional, el planteamiento del problema y la pregunta de investigación que se deriva de éste tienen un rol central. Sin un problema claramente planteado no tendríamos investigación, por lo que es común escuchar referirse a este como el corazón de la investigación. En general, el problema debe estar planteado de tal manera que nos invite a buscarle una respuesta. Para ello, las preguntas que nos hacemos son cruciales porque nos proporcionan los puntos esenciales de la ruta crítica que debemos seguir para la determinación de los referentes teóricos en los que nos apoyamos, las estrategias metodológicas que adoptamos y el tipo de análisis y reflexiones que realizamos.

Diversos autores coinciden en que la inspiración para las preguntas que nos hacemos en la ISPD surge de nuestra experiencia como docentes (Conchran-Smith \& Donnell, 2006; Hutchings, 2000), pero también insisten en que esta inspiración debe partir de las motivaciones más profundas del docente con su estudio, es decir, de la preocupación auténtica del docente por el aprendizaje de los estudiantes. En este sentido, Hutchings (2000) indica que la formulación de una buena pregunta para la ISPD no es sólo una tarea práctica e intelectual, sino que a menudo implica una cuestión moral y ética. Con ello no sólo se establece la necesidad de obtener un conocimiento, sino que se asume una responsabilidad con nuestra propia práctica docente al cuestionarla con la intención de superarla. De esta manera, el conocimiento cobra mayor valor al ser puesto desde el principio al servicio de los estudiantes y su aprendizaje. En este aspecto, Gilpin (2011) destaca que parte de la reflexión que acompaña a estas preguntas debe estar dirigida a hacernos más conscientes de nuestra propia responsabilidad como docentes hacia otros: los estudiantes.

La inquietud que surge a partir de lo anterior tiene que ver con lo que deberíamos cuestionarnos sobre nuestra práctica. En el libro llamado Opening Lines: Approaches to the Scholarship of Teaching and Learning, Pat Hutchings (2000), basada en los trabajos que se realizan en el Carnegie Academy for the Scholarship of Teaching and Learning (CASTL), se refiere a una taxonomía de las preguntas que usualmente se utilizan en la ISPD. En su clasificación, Hutchings distingue cuatro tipos de preguntas:

Investigación sobre la Prácica Docente en las Universidades, Martha Baéz

Cuaderno de Pedagogía Universitaria Año 9/ N. 17 /enero - junio 2012 / Santiago, Rep. Dom.: PUCMM / p. 3-8 
- $\quad$ El tipo de pregunta sobre ¿Qué funciona? - Estas indagaciones buscan evidencia de la efectividad relativa de distintos abordajes de enseñanza. Un tipo de pregunta que cabría dentro de esta categoría es, por ejemplo, cuestionarnos si los estudiantes de una clase conducida a través de la plataforma virtual Moodle están aprendiendo más que aquellos que la reciben de manera presencial.

- $\quad$ El tipo de pregunta sobre ¿Qué es? - Las preguntas propias de esta clasificación buscan describir las características que determinan un enfoque de enseñanza. De esta manera, más que tener la intención de evaluar un enfoque de enseñanza, estas preguntas buscan describir dicho enfoque con la intención de saber cómo aprenden los estudiantes con dicho enfoque. Hutchings identifica una investigación de la profesora Sherry Linckon -presentada en la obra antes citada- como ejemplo de este tipo de pregunta. La profesora Linckon quería comprender la experiencia de los cursos interdisciplinarios desde el punto de vista de los mismos estudiantes, el cual es un abordaje distinto al de comprenderlo desde el punto de vista de los docentes.

- El tipo de pregunta sobre la visión de lo posible - Son preguntas relacionadas con objetivos de enseñanza y aprendizaje que aún no se han cumplido u objetivos que son nuevos para el docente que se hace estas preguntas. Como ejemplo de esta categoría de pregunta, Hutchings cita el trabajo de una profesora de sociología que inicia su investigación preocupada por saber cómo sus estudiantes comprenden y se comprometen con el proceso de teorizar en contraposición a saber sobre sus conocimientos de teorías en particular. Este trabajo, eventualmente fue evolucionando hasta abordar las dimensiones emocionales del aprendizaje.

- El tipo de pregunta para la construcción de teorías - Estas preguntas están diseñadas para construir marcos teóricos en la ISPD, similares a los marcos utilizados en otras disciplinas. Un ejemplo de ello es la investigación de una profesora de inglés que ante las dificultades de algunos estudiantes para comprender un texto en particular aprovechó, no para identificar un problema de aprendizaje, sino para comprender lo que es esencial para aprender. En este sentido, la profesora no perseguía tanto la exploración de un aspecto de la práctica, sino la formulación de un nuevo marco conceptual para la formación del pensamiento sobre esa práctica.

Existen otras tipologías de preguntas para la ISPD, como las de Hubball y Clarke (2010), quienes consideran que las preguntas de investigación generalmente parten de una problematización de nuestra propia práctica institucional, curricular o en el aula. Estos autores las clasifican en cuatro tipos: 1) preguntas de contexto, que se enfocan en las estructuras educativas; 2 ) preguntas sobre procesos, orientadas a evaluar periódicamente asuntos de importancia para la formación; 3) preguntas de impacto, enfocadas en los resultados como consecuencia de una iniciativa educativa; 4) preguntas de seguimiento, relacionadas con temas de importancia que son el resultado de un impacto a largo plazo de las iniciativas educativas.
En definitiva, las preguntas de investigación que nos hacemos tienen una importancia central tanto porque nos ayudan a delimitar nuestro estudio como porque nos sitúan en un campo profesional y de conocimiento. Las anteriores son tipos de preguntas que nos empiezan a situar dentro de este fenómeno de la ISPD, los cuales requieren un abordaje especial para lograr su cometido.

\section{Métodos de investigación}

Al igual que en la investigación tradicional, en la ISPD el método es determinado por la pregunta de investigación que nos hemos formulado y el contexto en el que se desarrolla la investigación. Esto convierte la selección de los métodos en una decisión práctica y estratégica para responder a unas necesidades específicas.

Las posibilidades metodológicas para la ISPD son bastante amplias y, tal como indican Hubball y Clarke (2010), en ella se consideran abordajes tan variados como la investigación experimental, la investigación-acción, el estudio de caso, la teoría fundamentada, la etnografía en el aula, el análisis de la implementación, los estudios fenomenológicos, la investigación de desarrollo/evaluación de programas, la encuesta, las investigaciones longitudinales, entre otras. De igual manera, también se cuenta con una diversidad de métodos de recolección de información que pueden incluir grabaciones en video, cuestionarios, observación en el aula, grupos focales, entrevistas etnográficas, revisión de los planes de estudio/ programas de clases, revisión de los trabajos de los estudiantes, diario de campo, portafolios, entre otros.

A pesar de la variedad de métodos a nuestra disposición para conducir la ISPD, la experiencia de los estudiosos de esta práctica ha mostrado que en la ISPD el método está comúnmente determinado por la disciplina de la que proviene el docente (Hubball \& Clarke, 2010; Hutchings, 2000). Algunas de las explicaciones ante la limitación que se impone el docente en la decisión metodológica que adopta desde sus experiencias, tienen que ver con que éste se siente más confiado utilizando métodos conocidos y más intimidado y retado frente a los métodos que desconoce (Hutchings, 2000). Como es de suponerse, el riesgo de diseñar una metodología de investigación a partir de los métodos conocidos radica en la posibilidad de no considerar el más apropiado para la investigación que estamos planteando.

Lo ideal es que a la hora de decidir sobre nuestra metodología de investigación, consideremos una mezcla de métodos en lugar de un abordaje único. Esto es precisamente lo que nos recomienda Hutching (2000) cuando valora que la utilización de métodos mixtos siempre nos proporcionará información más útil que la utilización de un único método.

\section{El investigador en la ISPD}

La realidad del docente como investigador de su propia práctica nos lleva a una reflexión en dos sentidos. Por un lado, está la cuestión de las competencias del docente para conducir su propia ISPD. Por otro lado, se encuentra la cuestión del ejercicio individual y colectivo de esta práctica y la forma en que busca su legitimidad.

Investigación sobre la Prácica Docente en las Universidades, Martha Baéz

Cuaderno de Pedagogía Universitaria Año 9/ N. 17 /enero - junio 2012 / Santiago, Rep. Dom.: PUCMM / p. 3-8 
A esta altura es posible que nos hayamos hecho la siguiente pregunta: ¿es posible que cualquier docente interesado en mejorar su práctica docente se embarque en la ISPD? Esta es una preocupación común entre los que reflexionan sobre esta práctica (Conchran-Smith \& Donnell, 2006; Hubball \& Clark, 2010; Hutchings, 2000; Shulman, 2011). En este sentido, lo que generalmente preocupa saber es qué tanto necesita conocer el docente de su propia disciplina y qué tanto de las teorías y métodos de la investigación educativa como para poder conducir una ISPD.

En realidad, la ISPD no debería verse como un ejercicio separado de teoría y método. Al igual que en la investigación tradicional, la ISPD debería ser considerada como un híbrido que se sitúa en la intersección de la disciplina y la profesión (Hubball \& Clark, 2010). De esta manera, es importante conocer tanto las teorías fundamentales de pedagogía aplicada a nuestras disciplinas profesionales como tener nociones de los métodos y las técnicas más adecuadas para conducir nuestro estudio.

En otro sentido, ya hemos indicado anteriormente que una de las características de la ISPD es que el sujeto de estudio es el propio investigador. De esta manera, el docente no mira la realidad desde fuera, sino que se convierte en protagonista crítico y reflexivo de su propia práctica de enseñanza y de cómo ésta afecta el aprendizaje de sus estudiantes. Como es de suponerse, esta dualidad del docente como investigador y sujeto de estudio en un mismo escenario, necesariamente crea escepticismo sobre los resultados derivados de este tipo de prácticas.

Sin embargo, la ISPD no es sólo un ejercicio individual con el que nos comprometemos a enriquecer nuestra práctica. Aunque esto último es totalmente cierto, la ISPD también es un compromiso que es asumido colectivamente, en el que los colegas reflexionamos unos con otros, siempre con la intención de mejorar el aprendizaje de los estudiantes a partir de la transformación de nuestra docencia. Sobre esta construcción colectiva, Gilpin y Liston (2009) tuvieron la oportunidad de revisar cientos de trabajos relacionados con la ISPD, una de las conclusiones de esta revisión los llevó a afirmar que la ISPD y las actividades que a través de esta se promueven pueden llevar a una reconceptualización de la enseñanza y el aprendizaje como un esfuerzo compartido que transforma no sólo la enseñanza y el aprendizaje, sino nuestra relación entre unos y otros y de nosotros mismos con nuestro mundo.

La ISPD implica entonces tanto el conocimiento de la teoría como de los métodos de investigación pertinentes y es un ejercicio tanto individual como colectivo.

\section{Validez de la Investigación sobre la Práctica Docente}

Hasta el año pasado, los académicos más representativos de la ISPD en la educación superior se referían a ésta como un movimiento en proceso de construcción (Hutchings, Huber \& Ciccone, 2011). Esto de alguna manera refuerza la idea de que, aunque la ISPD se ha estado consolidando en las Instituciones de Educación Superior
(IES) anglosajonas y de Asia como una actividad generadora de conocimiento de valor para mejorar la enseñanza-aprendizaje, a ésta aun le falta un importante camino que recorrer para garantizar la validez científica del conocimiento que genera.

Sin embargo, para Shulman (2011), la ISPD garantiza su lugar como un enfoque investigativo a través de su búsqueda por hacerse pública, lo que implica exponerse a la revisión y evaluación de pares y hacerse accesible para el intercambio y uso de otros miembros de la propia comunidad de la disciplina a la que pertenece. En este sentido, como cualquier investigación, la ISPD se convierte en una propiedad comunitaria que es consumida y permite ser transformada. Esto tiene un gran valor para su aceptación porque, al abrirse a la crítica, permite que se refuercen y refinen sus conclusiones y seamos más asertivos en lograr el fin último de la ISPD, que es mejorar la calidad del aprendizaje.

Sin embargo, existen importantes argumentos que cuestionan la validez de la ISPD, algunos de los cuales provienen incluso de los mismos promotores de esta práctica. Dichas críticas han sido organizadas por Cochran-Smith y Donnell (2006) alrededor de cuatro temas importantes:

- Cuestionamientos sobre el conocimiento. Los críticos argumentan que existe un conocimiento formal, es decir teórico, respecto a lo que es enseñanza y aprendizaje, que se distingue de otro tipo de conocimiento que es más práctico, personal y experiencial. En este sentido, se cuestiona la capacidad de la ISPD de generar conocimiento, a menos que se realice desde las mismas convenciones, estrategias y garantías epistemológicas a las que se asocia al conocimiento formal.

- Cuestionamientos a los métodos. Debido a que el docente como sujeto de estudio es el mismo investigador y que éste proviene de áreas disciplinarias muy variadas, algunos cuestionan la capacidad que puede poseer para manejar las herramientas, mantener la distancia o realizar los análisis necesarios para conducir este tipo de investigación. Sin embargo, lograr trascender el propio ser para convertirse en investigador parece ser la crítica más aguda sobre este asunto.

- Cuestionamientos sobre la capacidad científica. Aunque relacionadas con los dos tipos de críticas anteriores, aquí se cuestiona más directamente el rigor científico de la ISPD. Este rigor se relaciona con la capacidad de generalización de las evidencias, lo que depende de las comparaciones entre distintos contextos académicos, tamaños significativos de las muestras y procedimientos y medidas uniformes. En este orden, Duffy (2000) también cuestiona la calidad de los datos que se levantan bajo esta modalidad de investigación en cuanto a que considera que cuando los docentes exponen a los estudiantes a un aprendizaje auténtico, pierden el control, lo que hace la evaluación de sus aprendizajes mucho más difícil de evidenciar.

- Cuestionamientos políticos. Estas críticas se vinculan con el fin político de la ISPD y sus bases ideológicas. Las críticas surgen en dos direcciones diametralmente opuestas, por un lado se critica la 
ISPD que no está conectada con la esfera política en cuanto a que no aborda temas relacionados con el poder, la equidad y los accesos. Por otro lado, existen críticas que también cuestionan la presencia de estas consideraciones en el entendido de que la investigación es y debe ser apolítica, neutral, no-ideológica y libre de valores.

- Una crítica al desarrollo personal y profesional. Finalmente, existen cuestionamientos que se relacionan con el hecho de que la ISPD se ha conceptualizado como un vehículo para el desarrollo personal y profesional de manera individual en lugar de considerársele como un modo de generar conocimiento o criticar la docencia. La crítica en este sentido es hacia el enfoque instrumental de esta práctica y su sostenimiento tácito del status quo en la academia. Por otro lado, también han surgido críticas al enfoque tan personal con el que se conduce la ISPD, acusándola de centrarse en el ego y la autocomplacencia. Los críticos argumentan que la investigación debe ser mucho más que un autodescubrimiento, ella debe incluir evidencia concreta de que los asuntos más serios y difíciles relacionados con la enseñanza, el aprendizaje y la educación se han enfrentado explícitamente.

Para los defensores de la ISPD, todas esas críticas que se relacionan tanto con problemas de tipo epistemológico y metodológico, como de tipo político y profesional, tienen sus raíces en que, al valorar la ISPD, sus críticos utilizan la misma mirada con la que ven la investigación tradicional. Sin lugar a dudas, la ISPD está de alguna manera retando los formatos de investigación tradicional con su provocativa propuesta para investigar, construir conocimiento y ejercer la docencia. Por ello, un punto de partida para superar estas críticas que nos sugieren los especialistas en el tema es comenzar por considerar la ISPD como un enfoque emergente de investigación distinto a la investigación tradicional, lo que requiere que nos despojemos de muchas de las convenciones con las que abordamos la investigación tradicional (Conchran-Smith \& Donnell, 2006).

\section{Importancia de la ISPD}

Entonces, a pesar de las críticas antes explicitadas, ¿por qué continua siendo importante la ISPD? Para Prosser (2008), su importancia radica en la interacción de lo genérico y lo contextual. De esta manera, la investigación tradicional produce descripciones y formas de pensar generales a las que la ISPD procura darle un significado particular en nuestro propio contexto y con nuestras propias prácticas de enseñanza-aprendizaje.

Por otro lado, para Shulman (2000), profesor emérito de la Escuela de Educación en la Universidad de Stanford, existen al menos tres razones para abogar por una seria inversión en la ISPD, a las que él se refiere como las tres $\mathrm{P}$ : profesionalismo, pragmatismo y política. El razonamiento de Shulman alrededor del profesionalismo tiene que ver con la responsabilidad del docente universitario frente a la doble profesión que debe atender: la de su propia disciplina y su profesión como educador. En la intersección de ambas profesiones, dice Shulman, la ISPD nos ayuda a mejorar la comprensión sobre la enseñanza y el aprendizaje en nuestro propio campo disciplinario.
Por otra parte, la perspectiva pragmática tiene que ver con la ayuda que nos proporciona la ISPD para guiar nuestros esfuerzos en diseñar y adaptar los procesos de enseñanza enfocados en el interés por mejorar el aprendizaje de los estudiantes. Finalmente, la perspectiva política apunta al valor de la ISPD como evidencia para la definición de políticas institucionales y para demostrar el compromiso asumido institucionalmente en el mejoramiento del aprendizaje de los estudiantes. En este sentido, la ISPD se convierte en una suerte de instrumento para la rendición de cuenta.

\section{Implicaciones de la ISPD para las políticas universitarias}

A medida que esta iniciativa por mejorar la enseñanza a través de la ISPD se va consolidando en las universidades y más docentes se comprometen con ella, los académicos que la promueven empiezan a querer comprender de manera más profunda las implicaciones de la ISPD a nivel institucional. En otras palabras, se empiezan a cuestionar sobre cómo la ISPD afecta las prácticas y políticas institucionales (Hutchings et al., 2011).

Después de que Shulman expusiera en el 2000 los argumentos antes mencionados para abogar por una inversión más seria en la ISPD, Hutchings et al. (2011) realizaron una encuesta procurando identificar las áreas específicas en las que la ISPD hacía una contribución importante a la práctica y política institucional. En esta encuesta participaron 59 instituciones de educación superior afiliadas al Carnegie Academy for Scholarship of Teaching and Learning (CASTL). A partir de los resultados de la misma, se identificaron cuatro áreas de importancia en las que la ISPD hace una contribución a nivel institucional.

- La forma en que los docentes manejan la enseñanza. La mayoría de los participantes en la encuesta indicaron que haber participado en la ISPD les permitió desarrollar nuevos enfoques de enseñanza en el aula. Los docentes sienten que tienen nuevas oportunidades para innovar y adquieren una especie de permiso para compartir ideas y aprender unos con otros, lo que no era común antes de esta práctica. Además, les llamó la atención la conciencia reflexiva con la que construyen los ambientes de aprendizaje que le ofrecen a los estudiantes, el nivel de atención que le prestan al aprendizaje y el involucramiento que poseen en temas relacionados con la educación en sus disciplinas, tanto dentro de los programas que conducen como en la institución en general.

- La forma en que se comprende y organiza el desarrollo profesional del docente. Esta fue la característica más mencionada entre los encuestados, quienes indicaron que el proceso de la ISPD ayuda a los estudiosos de la enseñanza y el aprendizaje a desarrollar sus capacidades como docentes practicantes, reflexivos e innovadores, al tiempo que hacer este trabajo público contribuye a un mayor conocimiento pedagógico en sus instituciones y en sus campos.

- La relación entre la investigación sobre la práctica docente y la evaluación institucional. Tanto la ISPD como la evaluación institucional comparten su enfoque en el aprendizaje de los 
estudiantes al abordar la calidad educativa, fundamentándose en evidencias sistemáticamente levantadas y en el compromiso de hacer público qué tanto están aprendiendo los estudiantes.

- La forma en que se valora y evalúa la labor de la enseñanza. Los involucrados en la encuesta aseguraban que, aunque la ISPD ha producido un cambio en la comprensión dominante de lo que se espera de los profesores como maestros, esta no necesariamente ha sido un elemento de valor para su retención, permanencia y promoción.

\section{Conclusión}

La ISPD es una especie de ejercicio de introspección que hacemos sobre nuestra propia enseñanza. Como propia debe entenderse tanto la enseñanza que realiza el docente como la que forma parte de la cultura institucional. Su condición de investigación viene dada por su fundamentación en evidencias concretas y por los esfuerzos que realiza para convertirse en propiedad comunitaria, haciendo públicos sus resultados, exponiéndolos a la crítica y al debate entre los colegas y ofreciéndolos para la reconstrucción de nuestra docencia individual y colectiva. Esto último se refiere a la docencia que ocurre en el aula y la que se relaciona con la cultura institucional.

Tanto la falta de madurez de esta práctica, como los retos que tiene por delante para resolver las críticas que la arropan, hacen que deba ser considerada como un fenómeno cuya definición y conceptualización continúa en construcción. Esto significa que las ISPD que emprendamos no deberían estar orientadas únicamente a responder las preguntas de investigación que las ocupan, sino también a aprender del proceso a través de los éxitos y retos que se enfrentan. De esta manera, estaremos también contribuyendo a alimentar el propio ejercicio de la ISPD.

Mientras tanto, la ISPD debe ser vista con una mirada distinta a la que usamos para ver la investigación tradicional. Ella es, en definitiva, una invitación a ejercer la docencia de una manera distinta, asumiendo la responsabilidad de comprender la dinámica de la enseñanza y el aprendizaje desde nuestras disciplinas y no dejar esta labor exclusivamente en manos de los especialistas de la educación.

La dinámica que implica la ISPD lleva a pensar que esta práctica, más que alimentar la experiencia institucional en investigación, lo que verdaderamente está alimentando es la docencia, al comprenderla y transformarla. De esta manera, la ISPD debe ser asumida tanto individual como colectivamente para que pueda ser sostenible. Debería ser parte de un esfuerzo institucional para motivar a los docentes a que sean mejores maestros y a desarrollar su profesión docente desde la comprensión profunda de su docencia a través de la ISPD.

\section{Referencias bibliográficas}

Cambridge, B. L. (Ed.) (2004). Campus progress: Supporting the scholarship of teaching and learning. Washington, DC: American Association for Higher Education.

Caster, B. \& Hautala, R. (2008). Changing Our Brains: Transforming a Traditional View of Scholarship and Teaching. International Journal for the Scholarship of Teaching and Learning, 2(2). Recuperado de http:// academics.georgiasouthern.edu/ijsotl/v2n2/essays_about_sotl/_ Caster/index.htm

Conchran-Smith, M. \& Donnell, K. (2006). Practioner Inquiry: Blurring the Boundaries of Research and Practice. En Green, J., Camilli, G. \& Elmore, P. (Ed.). Complementary Methods in Educacition Research. Washington, DC: American Educational Research Association (AERA). Mahwah, NJ: Lawrence Erlbaum Associates.

Duffy, D. (2000). Resilient Students, Resilient Communities. En Hutchings, P. (Ed.), Opening lines: approaches to the scholarship of teaching and learning (pp. 23-30). Menlo Park, CA: The Carnegie Foundation for the Advancement of Teaching.

Gilpin, L. \& Liston, D. (2009). Transformative Education in the Scholarship of Teaching and Learning: An Analysis of SoTL Literature. International Journal for the Scholarship of Teaching and Learning, 3(2). Recuperado de http://academics.georgiasouthern.edu/ijsotl/v3n2/articles/PDFs/ Article_GilpinListon.pdf

Gilpin, L. (2011). Scholarship of Teaching and Learning Trades. International Journal for the Scholarship of Teaching and Learning, 5(2). Recuperado de http://academics.georgiasouthern.edu/ijsotl/v5n1.html

Hubball, H. \& Clarke, A. (2010). Diverse methodological approaches and considerations for SoTL in Higher Education. The Canadian Journal for the Scholarship of Teaching and Learning, 1(1), art. 2. Recuperado de http://ir.lib.uwo.ca/cjsotl_rcacea/vol1/iss1/2/

Hutchings, P. (2000). Opening lines: approaches to the scholarship of teaching and learning. Menlo Park, CA: The Carnegie Foundation for the Advancement of Teaching.

Hutchings, P., Huber, M., \& Ciccone, A. (2011). Getting There: An integrative vision of the Scholarship of Teaching and Learning. International Journal for the Scholarship of Teaching and Learning, 5(1). Recuperado de http:// www.georgiasouthern.edu/ijsotl

McKinney, K. (s.f.). What is the Scholarship of Teaching and Learning (SoTL) in Higher Education. Recuperado de http://sotl.illinoisstate. edu/downloads/pdf/definesotl.pdf

Prosser, M. (2008). The scholarship of teaching and learning: what is it? A personal view. International Scholarship of Teaching and Learning, 2(2). Recuperado de http://academics.georgiasouthern.edu/ijsotl/v2n2/ invited_essays/_Prosser/index.htm

Shulman, L. (2000). From Minsk to Pinsk: why a scholarship of teaching and learning. The Journal Scholarship of Teaching and Learning, 1(1). Recuperado de http://www.fresnostate.edu/academics/csalt/documents/ Shulman2000.pdf

Shulman, L. (2011). The Scholarship of Teaching and Learning: A Personal Account and Reflection. International Journal for the Scholarship of Teaching and Learning, 5(1). Recuperado de http://academics. georgiasouthern.edu/ijsotl/v5n1.html 